\title{
Scalar extensions for algebraic structures of Eukasiewicz logic
}

\author{
Serafina Lapenta \\ Department of Mathematics, Computer Science and Economics, \\ University of Basilicata, Viale dell'Ateneo Lucano, 10 C.P. 85100, Potenza, Italy \\ serafina.lapenta@unibas.it \\ Ioana Leuştean \\ Department of Computer Science,
}

Faculty of Mathematics and Computer Science, University of Bucharest,

Academiei nr.14, sector 1, C.P. 010014, Bucharest, Romania

ioana@fmi.unibuc.ro

\begin{abstract}
In this paper we study the tensor product for MV-algebras, the algebraic structures of Łukasiewicz $\infty$-valued logic. Our main results are: the proof that the tensor product is preserved by the categorical equivalence between the MV-algebras and abelian lattice-order groups with strong unit and the proof of the scalar extension property for semisimple MValgebras. We explore consequences of this results for various classes of MV-algebras and lattice-ordered groups enriched with a product operation.
\end{abstract}

\section{Introduction}

MV-algebras are the algebraic counterpart of Łukasiewicz $\infty$-valued logic [6]. The variety of MV-algebras is generated by the standard model $\left([0,1], \oplus,{ }^{*}, 0\right)$, where $[0,1]$ is the unit real interval, $x \oplus y=\min \{1, x+y\}$ and $x^{*}=1-x$ for any $x, y \in[0,1]$. Since the interval $[0,1]$ is closed to the real product, a natural

problem was to analyze the systems obtained by enriching Łukasiewicz logic 
with a product operation. This investigation lead to fruitful investigations both in algebra and logic.

Several extension of the notion have been defined by endowing an MValgebra with products: an internal binary product leads to the notion of PMValgebra [9]; a scalar product leads to the notion of MV-module [10] and Riesz $\mathrm{MV}$-algebra [11]; a combination of both leads to the notion of $f \mathrm{MV}$-algebra [17]. For all these structures, corresponding logical systems are developed.

Within several important results, one main achievement in the theory is the categorical equivalence with abelian lattice-ordered groups with strong unit 20]. The categorical equivalence for MV-algebras extends naturally extended to any MV-algebra with product, and allows us to connect PMV-algebras, MVmodules, Riesz MV-algebras and $f \mathrm{MV}$-algebras with $\ell$-rings with strong unit, $\ell$-modules with strong unit, Riesz Spaces with strong unit and $f$-algebras with strong unit respectively.

The tensor product and its uses are well-known, therefore its definition was given by Martinez for lattice-ordered groups [18] and Mundici for MValgebras [21. An important subclass of MV-algebras are the semisimple ones which correspond, through the above mentioned categorical equivalence, to archimedean lattice-ordered structures. Since the tensor product does not preserve the semisimplicity, the semisimple tensor product was defined in [21] for MV-algebras and [16, 5] for lattice-ordered groups.

The scalar extension property (SEP) is one of the basic properties arising from a tensor product; while it is straightforward in the non-ordered case, it presents some difficulties in the framework of lattice-ordered structures. Note that MV-algebras have a natural order wich makes them lattice-ordered structures as well. In Section 3 we give an account of the results known so far, we prove SEP for the semisimple tensor product and we analyze some of its consequences. Since in Section 2 we prove that the tensor product is preserved by the categorical equivalence between MV-algebras and lattice-ordered groups, the results are stated both in the theory of MV-algebras and in the theory of lattice-ordered groups.

The scalar extension property led us to categorical adjunctions between semisimple MV-algebras and semisimple Riesz MV-algebras in Section 3 and between semisimple PMV-algebras and semisimple $f \mathrm{MV}$-algebras in Section 4 In Section $[5$ we sum up our results and provide an insight on their significance 
for Łukasiewicz logic.

\section{Preliminaries}

\subsection{MV-algebras and $\ell u$-groups}

An $M V$-algebra is an algebraic structure $\left(A, \oplus,{ }^{*}, 0\right)$, where $(A, \oplus, 0)$ is a commutative monoid, ${ }^{*}$ is an involution and the identity $\left(x^{*} \oplus y\right)^{*} \oplus y=\left(y^{*} \oplus x\right)^{*} \oplus x$ is satisfied for any $x, y, z \in A$. We further define $x \odot y=\left(x^{*} \oplus y^{*}\right)^{*}$ and $1=0^{*}$. An order can be defined on $A$ by setting $x \leq y$ if and only if $x^{*} \oplus y=0$; if we set $x \vee y=\left(x^{*} \oplus y\right)^{*} \oplus y$ and $x \wedge y=\left(x^{*} \vee y^{*}\right)^{*}$ then $(A, \vee, \wedge, 0,1)$ is a bounded distributive lattice. We refer to [8, 22] for all the unexplained notions concerning MV-algebras.

If $A$ is an MV-algebra we define a partial operation + as follows: for any $x, y \in A, x+y$ is defined if and only if $x \leq y^{*}$ and, in this case, $x+y=x \oplus y$. Note that this operation is cancellative. Assume $A, B$ and $C$ are MV-algebras. A function $\omega: A \rightarrow B$ is linear if $a \leq b^{*}$ implies $\omega(a) \leq \omega(b)^{*}$ and $\omega(a+b)=$ $\omega(a)+\omega(b)$. Bilinear functions $\beta: A \times B \rightarrow C$ are defined as usual.

Semisimple MV-algebras will play an important role in our development. If $A$ is an MV-algebra and $\operatorname{Rad}(A)$ is the intersection of its maximal ideals, then $A$ is semisimple if and only if $A$ is isomorphic to a separating MV-algebra of $[0,1]$-valued continuous functions defined over some compact Hausdorff space [8].

An $\ell u$-group is a pair $(G, u)$, where $G$ is an abelian lattice-ordered group [3, 2 ] and $u$ is a strong unit. If $(G, u)$ is an $\ell u$-group, then $[0, u]_{G}=\left([0, u], \oplus,{ }^{*}, 0\right)$ is an MV-algebra, where $[0, u]=\{x \in G \mid 0 \leq x \leq u\}$ and $x \oplus y=u \wedge(x+y)$, $x^{*}=u-x$ for any $x \in[0, u]$.

If $\mathbf{M V}$ is the category of MV-algebras and auG is the category of $\ell u$-groups equipped with morphisms that preserve the strong unit, then one defines a functor $\Gamma: \mathbf{a u G} \rightarrow \mathbf{M V}$ by $\Gamma(G, u)=[0, u]_{G}$ and $\Gamma(h)=\left.h\right|_{\left[0, u_{1}\right]_{G_{1}}}$, where $(G, u)$ is an $\ell u$-group and $h: G_{1} \rightarrow G_{2}$ is a morphism in auG between $\left(G_{1}, u_{1}\right)$ and $\left(G_{2}, u_{2}\right)$. The functor $\Gamma$ establishes a categorical equivalence between auG

and MV [20]. Moreover, through $\Gamma$, semisimple MV-algebras correspond to archimedean $\ell$-groups.

In the following $\Gamma(\mathbb{R}, 1)$ will be simply denoted $[0,1]$ (depending on context, 
the MV-algebra structure will be tacitly assumed). By Chang's completeness theorem [7, the variety of MV-algebras is generated by $[0,1]$.

\subsection{MV-algebras endowed with a product operation}

Product MV-algebras (PMV-algebras for short) have been defined in [9] in the general case and in [19] an equivalent axiomatization was provide for the unital and commutative structures. A unital $P M V$-algebra is a structure $\left(P, \oplus, \cdot{ }^{*}, 0\right)$ such that $\left(P, \oplus,{ }^{*}, 0\right)$ is an MV-algebra and $\cdot: P \times P \rightarrow P$ is a bilinear function such that $a \cdot(b \cdot c)=(a \cdot b) \cdot c$ and $a \cdot 1=1 \cdot a=a$ for any $a, b, c \in P$.

A further extension of the notion of MV-algebra has been introduced in [10]. If $P$ is a PMV-algebra, then an $M V$-module over $P(P-M V$-module $)$ is a structure $\left(M, \oplus,{ }^{*},\{\alpha \mid \alpha \in P\}, 0\right)$ such that $\left(M, \oplus,{ }^{*}, 0\right)$ is an MV-algebra, $\{\alpha \mid \alpha \in P\}$ is a family of unary operations such that the function $(\alpha, x) \mapsto \alpha x$ is bilinear, $(\alpha \cdot \beta) x=\alpha(\beta x)$ and $1 x=x$ for any $\alpha, \beta \in P$ and any $x \in M$.

Note that [10, Section 6.4] provides an equational characterization for these structures. Most important for our development is the case $P=[0,1]$. The MV-modules over [0,1] are called Riesz MV-algebras are studied in [11].

Finally, unital $f M V$-algebras have been introduced in [17] and they are algebraic structures $\left(A, \oplus,{ }^{*}, \cdot,\{\alpha\}_{\alpha \in[0,1]}, 0\right)$ such that $\left(A, \oplus,{ }^{*}, \cdot, 0\right)$ is a unital PMV-algebra, $\left(A, \oplus,{ }^{*},\{\alpha\}_{\alpha \in[0,1]}, 0\right)$ is a Riesz MV-algebra and the condition $\alpha(x \cdot y)=(\alpha x) \cdot y=x \cdot(\alpha y)$ is satisfied for any $\alpha \in[0,1]$ and $x, y \in A$.

We defined a hierarchy of algebraic structures, all of them having an MValgebra reduct. Hence there are forgetful functors from the categories PMV of PMV-algebras, RMV of Riesz MV-algebras and $\mathbf{f M V}$ of $f \mathrm{MV}$-algebras to MV. For each of this structures one can prove a categorical equivalence with an appropriate class of unital lattice-ordered structures having a lattice-ordered group reduct with a strong unit [9, 11, 17]. If $\mathbf{u R}$ is the category of unital $f$ - rings with strong unit ( $f u$-rings), $\mathbf{u R S}$ is the category of Riesz spaces with strong unit and fuAlg is the category of unital $f$-algebras with strong unit ( $f u$-algebras), then the categorical equivalence are presented in the following diagram, in which all horizontal arrows are suitable forgetful functors. 


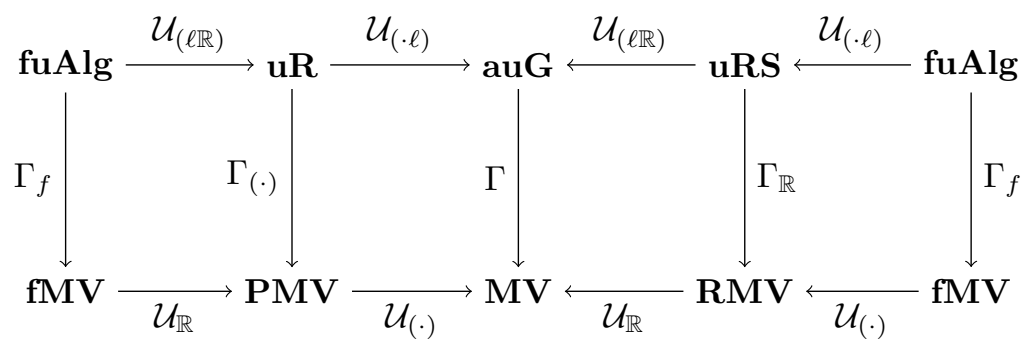

Figure 1.

Note that for the objects in $\mathbf{u R}$ and fuAlg have a unital ring reduct and we ask, in addition, that the ring-unity coincides with the strong unit. For the general theory of $\ell$-rings and $f$-rings, Riesz spaces, $\ell$-algebras and $f$-algebras we refer to [2, 3, 4, 5, 24].

We note that a PMV-algebra (Riesz MV-algebra, $f$ MV-algebra) is semisimple if its MV-algebra reduct is a semisimple MV-algebra and that semisimple PMV-algebras (semisimple Riesz MV-algebras, semisimple $f \mathrm{MV}$-algebras) correspond to archimedean $\ell$-rings (archimedean unital Riesz spaces, archimedean $f u$-algebras). Also, any unital and semisimple PMV-algebra ( $f$ MV-algebra) is commutative by the general theory of $f$-rings ( $f$-algebras) [4, 24].

In a similar manner, the MV-modules are categorically equivalent to appropriate classes of $\ell$-modules with strong unit ( $\ell u$-rings). We refer to [23] for the general theory of $\ell$-modules. If $(R, u)$ is an $\ell u$-ring and $P \simeq \Gamma_{(\cdot)}(R, u)$ then the category of MV-modules over $P$ is equivalent to the category of $\ell u$-modules over $R[10$.

\subsection{The tensor product $\otimes_{M V}$ and the semisimple tensor product $\otimes$}

A bimorphism is a bilinear function that is $\vee$-preserving and $\wedge$-preserving in each component. Bimorphisms were defined in [21, where the additional requirement $\beta(1,1)=1$ was imposed. In the present approach we eliminate this restriction.

The interval algebra of $A$ is the MV-algebra $[0, a]=\{x \in A \mid 0 \leq x \leq a\}$, endowed with the following operations $x \oplus_{a} y=(x \oplus y) \wedge a, x^{* a}=x^{*} \odot a$ for any $x, y \in[0, a]\left[21\right.$. In the follow we will use the notation $[0, a] \leq_{i} A$ in order to say that $[0, a]$ is an interval algebra of $A$.

The MV-algebraic tensor product was defined in 21] as a universal bimorphism. We shall use in the sequel a slightly modified universal property proved 
in [14]. For two MV-algebras $A$ and $B$, let $A \otimes_{M V} B$ be the tensor product and $\beta_{A, B}: A \times B \rightarrow A \otimes_{M V} B$ the universal bimorphism. Then the following universal property holds:

for any $M V$-algebra $C$ and for any bimorphism $\beta: A \times B \rightarrow C$, there is a unique homomorphism of $M V$-algebras $\omega: A \otimes_{M V} B \rightarrow[0, \beta(1,1)] \leq_{i} C$ such that $\omega \circ \beta_{A, B}=\beta$.

For $a \in A$ and $B \in B$ we denote $a \otimes_{M V} b=\beta_{A, B}(a, b)$. Note that $A \otimes_{M V} B$ is generated by $\beta_{A, B}(A \times B)$.

In 21] the author proves that there exists a semisimple MV-algebra $A$ such that $A \otimes_{M V} A$ is not semisimple. Therefore he defines the semisimple tensor product of $A$ and $B$, semisimple MV-algebras, by

$$
A \otimes B=A \otimes_{M V} B / \operatorname{Rad}\left(A \otimes_{M V} B\right) .
$$

For the semisimple tensor product the universal property holds with respect to semisimple MV-algebras.

The following representation theorem is crucial for our development.

Theorem 1.1. [21] Let $A$ and $B$ be semisimple $M V$-algebras, and let $X, Y$ be the set such that $A \subseteq C(X)$ and $B \subseteq C(Y)$. Let $\gamma: A \times B \rightarrow C(X \times Y)$ be the map defined by $\gamma(a, b)(x, y)=a(x) b(y)$. Then $\gamma$ is a bimorphism and $A \otimes B$ is isomorphic to the $M V$-subalgebra of $C(X \times Y)$ generated by $\gamma(a, b)$, with $a \in A$ and $b \in B$.

Few types of tensor products are defined in the literature of partially-ordered and lattice-ordered groups [18, 23]. We recall the one that will be used in the sequel.

Let $G, H$ and $L$ abelian lattice-ordered groups. An $\ell$-bilinear function is a map $\gamma: G \times H \rightarrow L$ such that $\gamma(x, \cdot)$ and $\gamma(\cdot, y)$ are homomorphisms of $\ell$-groups when $x$ and $y$ are positive elements in $G$ and $H$, respectively. Hence, the tensor product $G \otimes_{\ell} H$ and its universal $\ell$-bilinear function $\gamma_{G, H}: G \times H \rightarrow G \otimes_{\ell} H$ satisfy a universal property with respect to abelian lattice-ordered groups and $\ell$-bilinear functions. For $x \in G$ and $y \in H$ we denote $x \otimes_{\ell} y=\gamma_{G, H}(x, y)$.

In [5, 16] the authors provide a construction for the tensor product of archimedean $\ell$-groups, denoted by $\otimes_{a}$ and they prove the universal property with respect to archimedean structures. 


\section{The $\Gamma$-functor and the tensor product $\otimes_{M V}$}

In this section we prove that the functor $\Gamma:$ auG $\rightarrow \mathbf{M V}$ commutes with the tensor product, both in the general and in the archimedean case. To do this, we prove an extension result for bimorphisms.

As a preliminary step, we give the detailed proof of the fact that any factor of the MV-algebraic tensor product is embedded in the tensor product. If $A$ and $B$ are $\mathrm{MV}$-algebras, then we define

$$
\begin{gathered}
\iota_{A}: A \rightarrow A \otimes_{M V} B \text { and } \iota_{B}: B \rightarrow A \otimes_{M V} B \\
\iota_{A}(a)=a \otimes_{M V} 1_{B} \text { and } \iota_{B}(b)=1_{A} \otimes_{M V} b \text { for any } a \in A \text { and } b \in B .
\end{gathered}
$$

The functions $\iota_{A}$ and $\iota_{B}$ are embeddings of MV-algebras [13] (private communication). We sketch the proof, for the sake of completeness.

Proposition 2.1. The maps $\iota_{A}: A \rightarrow A \otimes_{M V} B$ and $\iota_{B}: B \rightarrow A \otimes_{M V} B$ defined as $\iota_{A}(a)=a \otimes_{M V} 1_{B}$ and $\iota_{B}(b)=1_{A} \otimes_{M V} b$ for any $a \in A$ and $b \in B$, are embeddings.

Proof. By [22, Theorem 2.20] there exists a MV-algebra $D$ such that both $A$ and $B$ embeds in it. By [8, Theorem 9.5.1] there exists a set $X$ and a MValgebra embedding $f: D \hookrightarrow\left({ }^{*}[0,1]\right)^{X}$, therefore there exist two embeddings

$A \stackrel{f_{A}}{\rightarrow}(*[0,1])^{X}$ and $B \stackrel{f_{B}}{\rightarrow}(*[0,1])^{X}$. We remark that $\left({ }^{*}[0,1]\right)^{X}$ is a unital and commutative PMV-algebra, therefore we define the following bimorphism

$\beta: A \times B \rightarrow\left({ }^{*}[0,1]\right)^{X}, \beta_{1}(a, b)=f_{A}(a) \cdot f_{B}(b)$ for any $a \in A$ and any $b \in B$. By the universal property in [21], there exists $\omega: A \otimes_{M V} B \rightarrow\left({ }^{*}[0,1]\right)^{X}$ such that $\omega\left(a \otimes_{M V} b\right)=f_{A}(a) \cdot f_{B}(b)$.

Assume that $\iota_{A}\left(a_{1}\right)=\iota_{A}\left(a_{2}\right)$, that is $a_{1} \otimes_{M V} 1_{B}=a_{2} \otimes_{M V} 1_{B}$, then $f_{A}\left(a_{1}\right)=$ $\omega\left(a_{1} \otimes_{M V} 1_{B}\right)=\omega\left(a_{2} \otimes_{M V} 1_{B}\right)=f_{A}\left(a_{2}\right)$. Since $f_{A}$ is an embedding, the conclusion follows. Analogously, $\iota_{B}$ is an embedding.

Assume $\left(G, u_{G}\right)\left(H, u_{H}\right)$ and $\left(L, u_{L}\right)$ are $\ell u$-groups and $\gamma: G \times H \rightarrow L$ is an $\ell$ - bilinear function. We say that $\gamma$ is $\ell u$-bilinear if $\gamma\left(u_{G}, u_{H}\right) \leq u_{L}$.

In the sequel we prove that a bimorphism uniquely extends to an $\ell u$-bilinear function.

Proposition 2.2. If $A=\Gamma\left(G, u_{G}\right), B=\Gamma\left(H, u_{H}\right)$ and $C=\Gamma\left(L, u_{L}\right)$ and $\beta: A \times B \rightarrow C$ is a bimorphism, then there exists an unique $\ell u$-bilinear function $\bar{\beta}: G \times H \rightarrow L$ that extends $\beta$. 
Proof. Let $a$ be a fixed element in $A$ and denote $\beta_{a}=\beta(a, \cdot)$. Hence, by [21, Proposition 2.3], we have $\beta_{a}: B \rightarrow[0, \beta(a, 1)] \leq_{i} C$ is a homomorphism of MV-algebras. By [14, Proposition 2.9], there exists a unique homomorphism of $\ell$-groups $\overline{\beta_{a}}$ such that $\overline{\beta_{a}}: H \rightarrow L$, and $\left.\overline{\beta_{a}}\right|_{B}=\beta_{a}$.

Step 1. We prove that the map $\gamma_{h}: A \rightarrow L$, defined by $\gamma_{h}(a)=\overline{\beta_{a}}(h)$ for any $h \in H$, is linear.

Let the sum $a_{1}+a_{2}$ be defined in $A$ and let $h \in H$, since $B$ generates the positive cone of $\left(H, u_{H}\right), h=h^{+}-h^{-}$, where $h^{+}=s_{1}+\ldots+s_{n}$ and $h^{-}=t_{1}+\ldots+t_{m}$, with $s_{i}, t_{j} \in B$ for any $i=1, \ldots, n$ and $j=1, \ldots, m$.

$$
\begin{gathered}
\gamma_{h}\left(a_{1}+a_{2}\right)=\overline{\beta_{a_{1}+a_{2}}}\left(\left(s_{1}+\ldots+s_{n}\right)-\left(t_{1}+\ldots+t_{m}\right)\right)= \\
\left(\overline{\beta_{a_{1}+a_{2}}}\left(s_{1}\right)+\ldots+\overline{\beta_{a_{1}+a_{2}}}\left(s_{n}\right)\right)-\left(\overline{\beta_{a_{1}+a_{2}}}\left(t_{1}\right)+\ldots+\overline{\beta_{a_{1}+a_{2}}}\left(t_{m}\right)\right)=\left(\beta\left(a_{1}+a_{2}, s_{1}\right)+\right. \\
\left.\ldots+\beta\left(a_{1}+a_{2}, s_{n}\right)\right)-\left(\beta\left(a_{1}+a_{2}, t_{1}\right)+\ldots \beta\left(a_{1}+a_{2}, t_{m}\right)\right)=\left(\beta\left(a_{1}, s_{1}\right)+\beta\left(a_{2}, s_{1}\right)+\right. \\
\left.\ldots+\beta\left(a_{1}, s_{n}\right)+\beta\left(a_{2}, s_{n}\right)\right)-\left(\beta\left(a_{1}, t_{1}\right)+\beta\left(a_{2}, t_{1}\right)+\ldots \beta\left(a_{1}, t_{m}\right)+\beta\left(a_{2}, t_{m}\right)\right)= \\
{\left[\left(\overline{\beta_{a_{1}}}\left(s_{1}\right)+\ldots+\overline{\beta_{a_{1}}}\left(s_{n}\right)\right)-\left(\overline{\beta_{a_{1}}}\left(t_{1}\right)+\ldots+\overline{\beta_{a_{1}}}\left(t_{m}\right)\right)\right]+\overline{\beta_{a_{1}}}(h)+\overline{\beta_{a_{2}}}(h)=} \\
\left.\left[\overline{\beta_{a_{2}}}\left(s_{1}\right)+\ldots+\overline{\beta_{a_{2}}}\left(s_{n}\right)\right)-\left(\overline{\beta_{a_{2}}}\left(t_{1}\right)+\ldots+\overline{\beta_{a_{2}}}\left(t_{m}\right)\right)\right]=\overline{\gamma_{h}\left(a_{1}\right)+\gamma_{h}\left(a_{2}\right) .}
\end{gathered}
$$

Step 2. $\gamma_{h}$ commutes with $\wedge$ and $\vee$.

We first remark that an element of $\left(H, u_{H}\right)$ is a good sequence according with Mundici construction of the inverse of the functor $\Gamma$. Moreover, by [1] and it is possible to take indexes in $\mathbb{Z}$ instead of quotients of sequence, then

$$
\begin{gathered}
\gamma_{h}\left(a_{1} \wedge a_{2}\right)=\overline{\beta_{a_{1} \wedge a_{2}}}(h)=\overline{\beta_{a_{1} \wedge a_{2}}}\left(\left(h_{i}\right)_{i \in \mathbb{Z}}\right)=\left(\overline{\beta_{a_{1} \wedge a_{2}}}\left(h_{i}\right)\right)_{i \in \mathbb{Z}}= \\
\left(\beta\left(a_{1} \wedge a_{2}, h_{i}\right)\right)_{i \in \mathbb{Z}}=\left(\beta\left(a_{1}, h_{i}\right) \wedge \beta\left(a_{2}, h_{i}\right)\right)_{i \in \mathbb{Z}}=\left(\beta\left(a_{1}, h_{i}\right)\right)_{i \in \mathbb{Z}} \wedge\left(\beta\left(a_{2}, h_{i}\right)\right)_{i \in \mathbb{Z}}= \\
\left(\overline{\beta_{a_{1}}}\left(h_{i}\right)\right)_{i \in \mathbb{Z}} \wedge\left(\overline{\beta_{a_{2}}}\left(h_{i}\right)\right)_{i \in \mathbb{Z}}=\overline{\beta_{a_{1}}}(h) \wedge \overline{\beta_{a_{2}}}(h)=\gamma_{h}\left(a_{1}\right) \wedge \gamma_{h}\left(a_{2}\right),
\end{gathered}
$$

and similarly for $\vee$.

Therefore the map $\gamma_{h}:\left[0, u_{G}\right] \rightarrow L$ is linear and commutes with $\vee$ and $\wedge$, i.e. $\gamma_{h}:\left[0, u_{G}\right] \rightarrow\left[0, \gamma_{h}\left(u_{G}\right)\right] \leq_{i}\left[0, u_{L}\right]$ is an homomorphism of MV-algebras [21, Proposition 2.3] and consequently, by [14, Proposition 2.9] there exists a unique homomorphism of $\ell$-groups $\overline{\gamma_{h}}: G \rightarrow K \leq L$, where $K$ is the $\ell$-group generated by $\gamma_{h}(A)$ and $\left.\overline{\gamma_{h}}\right|_{A}=\gamma_{h}$.

We define now $\bar{\beta}: G \times H \rightarrow L$ as $\bar{\beta}(g, h)=\overline{\gamma_{h}}(g)$. By the construction, $\bar{\beta}(\cdot, h)$ is a homomorphism of $\ell$-groups.

Step 3. $\bar{\beta}(g, \cdot)$, with $g$ fixed element in $G$, is linear.

Let $h_{1}, h_{2}$ be elements in $H$; there exist suitable elements in the unit interval such that $g^{+}=s_{1}+\ldots+s_{n}$ and $g^{-}=t_{1}+\ldots+t_{m}$. 


$$
\begin{gathered}
\bar{\beta}\left(g, h_{1}+h_{2}\right)=\overline{\gamma_{h_{1}+h_{2}}}(g)= \\
\left(\overline{\gamma_{h_{1}+h_{2}}}\left(s_{1}\right)+\ldots+\overline{\gamma_{h_{1}+h_{2}}}\left(s_{n}\right)\right)-\left(\overline{\gamma_{h_{1}}+h_{2}}\left(t_{1}\right)+\ldots+\overline{\gamma_{h_{1}+h_{2}}}\left(t_{m}\right)\right)= \\
\left.\left(\overline{\beta_{s_{1}}}\left(h_{1}+h_{2}\right)+\ldots+\overline{\beta_{s_{n}}}\left(h_{1}+h_{2}\right)\right)-\overline{\left(\overline{\beta_{t_{1}}}\right.}\left(h_{1}+h_{2}\right)+\ldots+\overline{\beta_{t_{m}}}\left(h_{1}+h_{2}\right)\right)= \\
\left(\overline{\beta_{s_{1}}}\left(h_{1}\right)+\overline{\beta_{s_{1}}}\left(h_{2}\right)+\ldots+\overline{\beta_{s_{n}}}\left(h_{1}\right)+\overline{\beta_{s_{n}}}\left(h_{2}\right)\right)-\left(\overline{\beta_{t_{1}}}\left(h_{1}\right)+\overline{\beta_{t_{1}}}\left(h_{2}\right)+\ldots+\right. \\
\left.\overline{\beta_{t_{m}}}\left(h_{1}\right)+\overline{\beta_{t_{m}}}\left(h_{2}\right)\right)=\left[\left(\gamma_{h_{1}}\left(s_{1}\right)+\ldots+\gamma_{h_{1}}\left(s_{n}\right)\right)-\left(\gamma_{h_{1}}\left(t_{1}\right)+\ldots+\gamma_{h_{1}}\left(t_{m}\right)\right)\right]+ \\
{\left[\left(\gamma_{h_{2}}\left(s_{1}\right)+\ldots+\gamma_{h_{2}}\left(s_{n}\right)\right)-\left(\gamma_{h_{2}}\left(t_{1}\right)+\ldots+\gamma_{h_{2}}\left(t_{m}\right)\right)\right]=\overline{\gamma_{h_{1}}}(g)+\overline{\gamma_{h_{2}}}(g)=} \\
\bar{\beta}\left(g, h_{1}\right)+\bar{\beta}\left(g, h_{2}\right) .
\end{gathered}
$$

Step 4. $\bar{\beta}(g, \cdot)$ commute with $\vee$ and $\wedge$.

We will use again good sequences

$$
\begin{gathered}
\bar{\beta}\left(g, h_{1} \wedge h_{2}\right)=\overline{\gamma_{h_{1} \wedge h_{2}}}(g)=\overline{\gamma_{h_{1} \wedge h_{2}}}\left(\left(g_{i}\right)_{i \in \mathbb{Z}}\right)=\left(\overline{\gamma_{h_{1} \wedge h_{2}}}\left(g_{i}\right)\right)_{i \in \mathbb{Z}}= \\
\left(\gamma_{h_{1} \wedge h_{2}}\left(g_{i}\right)\right)_{i \in \mathbb{Z}}=\left(\overline{\beta_{g_{i}}}\left(h_{1} \wedge h_{2}\right)\right)_{i \in \mathbb{Z}}=\left(\overline{\beta_{g_{i}}}\left(h_{1}\right) \wedge \overline{\beta_{g_{i}}}\left(h_{2}\right)\right)_{i \in \mathbb{Z}}=\left(\overline{\beta_{g_{i}}}\left(h_{1}\right)\right)_{i \in \mathbb{Z}} \wedge \\
\left.\left(\overline{\beta_{g_{i}}}\left(h_{2}\right)\right)_{i \in \mathbb{Z}}=\left(\overline{\gamma_{h_{1}}}\left(g_{i}\right)\right)_{i \in \mathbf{Z}} \wedge \overline{\gamma_{h_{2}}}\left(g_{i}\right)\right)_{i \in \mathbf{Z}}=\overline{\gamma_{h_{1}}}(g) \wedge \overline{\gamma_{h_{2}}}(g)=\bar{\beta}\left(g, h_{1}\right) \wedge \bar{\beta}\left(g, h_{2}\right) .
\end{gathered}
$$

The same can be done for $\vee$ and $\bar{\beta}(g, \cdot)$ is a homomorphism of $\ell$-groups. Moreover, $\bar{\beta}\left(u_{G}, u_{H}\right)=\beta\left(u_{G}, u_{H}\right) \leq u_{L}$.

In order to prove the uniqueness, we assume that $\tilde{\beta}: G \times H \rightarrow L$ is another $\ell u$-bilinear function that extends $\beta$. If $a \in A$ then $\tilde{\beta}(a, \cdot)$ is an extension of $\beta_{a}$, so it coincides with $\overline{\beta_{a}}$. It follows that $\bar{\beta}(a, \cdot): H \rightarrow L$ and $\tilde{\beta}(a, \cdot): H \rightarrow L$ coincide for any $a \in A$. By linearity, they coincide for any $g \in G$.

The main result of this section is Theorem 2.1, which asserts that the functor $\Gamma: \mathbf{a u G} \rightarrow \mathbf{M V}$ preserves the tensor product.

Note that, if $\left(G, u_{G}\right)$ and $\left(H, u_{H}\right)$ are $\ell u$-groups then $u_{G} \otimes_{\ell} u_{H}$ is strong unit in $G \otimes_{\ell} H$ [18, 3.6]. In the sequel we prove two preliminary lemmas.

Lemma 2.1. Let $\left(G, u_{G}\right),\left(H, u_{H}\right)$ and $\left(L, u_{L}\right)$ be lu-groups. For any bimorphism $\gamma: \Gamma\left(G, u_{G}\right) \times \Gamma\left(H, u_{H}\right) \rightarrow \Gamma\left(L, u_{L}\right)$ there is a unique homomorphism of $M V$-algebras $\omega: \Gamma\left(G \otimes_{\ell} H, u_{G} \otimes_{\ell} u_{H}\right) \rightarrow\left[0, \gamma\left(u_{G}, u_{H}\right)\right] \leq_{i} \Gamma\left(L, u_{L}\right)$ such that $\omega\left(x \otimes_{\ell} y\right)=\gamma(x, y)$ for any $x \in \Gamma\left(G, u_{G}\right)$ and $y \in \Gamma\left(H, u_{h}\right)$.

Proof. We set $A=\Gamma\left(G, u_{G}\right), B=\Gamma\left(H, u_{H}\right), C=\Gamma\left(L, u_{L}\right)$ and we suppose that $\gamma: A \times B \rightarrow C$ is a bimorphism. By Proposition 2.2, there is a $\ell u$-bilinear function $\widetilde{\gamma}: G \times H \rightarrow L$ which extends $\gamma$, so there is a unique homomorphism of $\ell$-groups $\widetilde{\omega}: G \otimes_{\ell} H \rightarrow L$ such that $\widetilde{\omega} \circ \gamma_{G, H}=\widetilde{\gamma}$.

It follows that $\widetilde{\omega}\left(u_{G} \otimes_{\ell} u_{H}\right)=\widetilde{\gamma}\left(u_{G}, u_{H}\right)=\gamma(1,1) \leq u_{L}$, therefore by [14, Lemma 2.8] the restriction $\omega: \Gamma\left(G \otimes_{\ell} H, u_{G} \otimes_{\ell} u_{H}\right) \rightarrow\left[0, \gamma\left(u_{G}, u_{H}\right)\right] \leq_{i} C$, defined by $\omega(\mathbf{x})=\widetilde{\omega}(\mathbf{x})$ for any $\mathbf{x} \in \Gamma\left(G \otimes_{\ell} H, u_{G} \otimes_{\ell} u_{H}\right)$, is a homomorphism 
of MV-algebras and $\omega\left(x \otimes_{\ell} y\right)=\widetilde{\omega}\left(x \otimes_{\ell} y\right)=\left(\widetilde{\omega} \circ \gamma_{G, H}\right)(x, y)=\widetilde{\gamma}(x, y)=\gamma(x, y)$, for any $x \in \Gamma\left(G, u_{G}\right)$ and $y \in \Gamma\left(H, u_{H}\right)$.

In order to prove the uniqueness, let $\theta: \Gamma\left(G \otimes_{\ell} H, u_{G} \otimes_{\ell} u_{H}\right) \rightarrow\left[0, \gamma\left(u_{G}, u_{H}\right)\right] \leq_{i}$ $C$ be another homomorphism of MV-algebras such that $\theta\left(x \otimes_{\ell} y\right)=\gamma(x, y)$ for any $x \in \Gamma\left(G, u_{G}\right)$ and $y \in \Gamma\left(H, u_{H}\right)$. By [14, Proposition 2.9] there is a unique homomorphism of $\ell$-groups $\widetilde{\theta}: G \otimes H \rightarrow L$ such that $\left.\widetilde{\theta}\right|_{\Gamma\left(G \otimes \ell H, u_{G} \otimes_{\ell} u_{H}\right)}=\theta$. If $\tau$ is defined as $\tilde{\theta} \circ \gamma_{G, H}$ then is straightforward that $\tau: G \times H \rightarrow L$ is a $\ell u$-bilinear function and $\tau(x, y)=\theta(x \otimes y)=\gamma(x, y)$. Since $\widetilde{\gamma}$ is the unique $\ell u$-bilinear function that extends $\gamma$ we get $\tau=\widetilde{\gamma}$ and $\widetilde{\theta} \circ \gamma_{G, H}=\widetilde{\gamma}$. It follows that $\widetilde{\theta}=\widetilde{\omega}$ and $\theta=\omega$.

In the following, we introduce a notation. Let $A, B$ be MV-algebras and $\left(G_{A}, u_{A}\right),\left(G_{B}, u_{B}\right)$ lu-groups such that $A \simeq \Gamma\left(G_{A}, u_{A}\right)$ and $B \simeq \Gamma\left(G_{B}, u_{B}\right)$. Assume $\left\{\eta_{A}\right\}_{A \in \mathbf{M V}}$ is the natural isomorphism between the categories $\mathbf{M V}$ and auG, i.e. $\eta_{A}: A \rightarrow \Gamma\left(G_{A}, u_{A}\right)$ and $\eta_{B}: B \rightarrow \Gamma\left(G_{B}, u_{B}\right)$ are isomorphisms of MV-algebra. Hence we define $\gamma_{A, B}: A \times B \rightarrow \Gamma\left(G_{A} \otimes_{\ell} G_{B}, u_{A} \otimes_{\ell} u_{B}\right)$ by $\gamma_{A, B}(x, y)=\eta_{A}(x) \otimes_{\ell} \eta_{B}(y)$ for any $x \in A$ and $y \in B$.

Lemma 2.2. Let $A, B$ and $\left(G_{A}, u_{A}\right),\left(G_{B}, u_{B}\right)$ lu-groups such that $A \simeq$ $\Gamma\left(G_{A}, u_{A}\right)$ and $B \simeq \Gamma\left(G_{B}, u_{B}\right)$. For any $M V$-algebra $C$ and any bimorphism $\gamma: A \times B \rightarrow C$ there is a unique homomorphism of $M V$-algebras $\omega: \Gamma\left(G_{A} \otimes_{\ell}\right.$ $\left.G_{B}, u_{A} \otimes_{\ell} u_{B}\right) \rightarrow\left[0, \gamma\left(1_{A}, 1_{B}\right)\right] \leq_{i} C$ such that $\omega \circ \gamma_{A, B}=\gamma$.

Proof. In the following $A \otimes_{\ell} B$ will denote the MV-algebra $\Gamma\left(G_{A} \otimes_{\ell} G_{B}, u_{A} \otimes_{\ell}\right.$ $\left.u_{B}\right)$. Suppose that $C$ is an arbitrary MV-algebra and $\gamma: A \times B \rightarrow C$ is a bimorphism. We define $\gamma_{1}: \Gamma\left(G_{A}, u_{A}\right) \times \Gamma\left(G_{B}, u_{B}\right) \rightarrow \Gamma\left(G_{C}, u_{c}\right)$ by

$$
\gamma_{1}(x, y)=\eta_{C}\left(\gamma\left(\eta_{A}^{-1}(x), \eta_{B}^{-1}(y)\right)\right) \text {. }
$$

Since $\eta_{C}, \eta_{A}^{-1}$ and $\eta_{B}^{-1}$ are MV-algebra isomorphisms, $\gamma_{1}$ is also a bimorphism, and by Lemma 2.1, there is a unique homomorphism of MV-algebras $\omega_{1}$ : $\Gamma\left(G_{A} \otimes_{\ell} G_{B}, u_{A} \otimes_{\ell} u_{B}\right) \rightarrow\left[0, \gamma_{1}\left(u_{A}, u_{B}\right)\right] \leq_{i} \Gamma\left(G_{C}, u_{C}\right)$ such that $\omega_{1}\left(x \otimes_{\ell} y\right)=$ $\gamma_{1}(x, y)$ for any $x \in \Gamma\left(G_{A}, u_{A}\right)$ and $y \in \Gamma\left(G_{B}, u_{B}\right)$. Remark that the definition domain of $\omega_{1}$ is $A \otimes_{\ell} B$. Hence, if we define $\omega(\mathbf{x})=\eta_{C}^{-1}\left(\omega_{1}(\mathbf{x})\right)$ for any $\mathbf{x} \in$ $A \otimes_{\ell} B$, by [14, Lemma 2.8] $\omega: A \otimes_{\ell} B \rightarrow\left[0, \eta_{C}^{-1}\left(\gamma_{1}\left(u_{A}, u_{B}\right)\right)\right] \leq_{i} C$ is a homomorphism of MV-algebras. We have $\eta_{C}^{-1}\left(\gamma_{1}\left(u_{A}, u_{B}\right)\right)=\gamma\left(\eta_{A}^{-1}\left(u_{A}\right), \eta_{B}^{-1}\left(u_{B}\right)\right)=$ $\gamma\left(1_{A}, 1_{B}\right)$.

For any $x \in A$ and $y \in B$ it follows that

$$
\left(\omega \circ \gamma_{A, B}\right)(x, y)=\omega\left(\eta_{A}(x) \otimes_{\ell} \eta_{B}(y)\right)=\eta_{C}^{-1}\left(\omega_{1}\left(\eta_{A}(x) \otimes_{\ell} \eta_{B}(y)\right)\right)=
$$




$$
=\eta_{C}^{-1}\left(\gamma_{1}\left(\eta_{A}(x), \eta_{B}(y)\right)\right)=\gamma(x, y) .
$$

Hence, $\omega \circ \gamma_{A, B}=\gamma$. In order to prove the uniqueness, suppose that $\theta: A \otimes_{\ell}$ $B \rightarrow\left[0, \gamma\left(1_{A}, 1_{B}\right)\right] \leq_{i} C$ is another homomorphism of MV-algebras such that $\theta \circ \gamma_{A, B}=\gamma$. Using the isomorphism $\eta_{C}$ we define the following homomorphism of MV-algebras: $\theta_{1}: A \otimes_{\ell} B \rightarrow\left[0, \eta_{C}\left(\gamma\left(1_{A}, 1_{B}\right)\right)\right] \leq \Gamma\left(G_{C}, u_{C}\right)$, with $\theta_{1}(\mathbf{x})=$ $\eta_{C}(\theta(\mathbf{x}))$, for any $\mathbf{x} \in A \otimes_{\ell} B$. Remark that

$$
\eta_{C}\left(\gamma\left(1_{A}, 1_{B}\right)\right)=\eta_{C}\left(\gamma\left(\eta_{A}^{-1}\left(u_{A}\right), \eta_{B}^{-1}\left(u_{B}\right)\right)\right)=\gamma_{1}\left(u_{A}, u_{B}\right) .
$$

Moreover, for any $x \in \Gamma\left(G_{A}, u_{A}\right)$ and $y \in \Gamma\left(G_{B}, u_{B}\right)$ we get

$$
\begin{aligned}
& \theta_{1}\left(x \otimes_{\ell} y\right)=\eta_{C}\left(\theta\left(x \otimes_{\ell} y\right)\right)=\eta_{C}\left(\theta\left(\eta_{A}\left(\eta_{A}^{-1}(x)\right) \otimes_{\ell} \eta_{B}\left(\eta_{B}^{-1}(y)\right)\right)\right)= \\
& =\eta_{C}\left(\theta\left(\gamma_{A, B}\left(\eta_{A}^{-1}(x), \eta_{B}^{-1}(y)\right)\right)\right)=\eta_{C}\left(\gamma\left(\eta_{A}^{-1}(x), \eta_{B}^{-1}(y)\right)\right)=\gamma_{1}(x, y) .
\end{aligned}
$$

It follows that $\theta_{1}$ satisfies the properties that uniquely characterize $\omega_{1}$ by Lemma 2.1, so $\theta_{1}=\omega_{1}$. In consequence,

$$
\omega(\mathbf{x})=\eta_{C}^{-1}\left(\omega_{1}(\mathbf{x})\right)=\eta_{C}^{-1}\left(\theta_{1}(\mathbf{x})\right)=\theta(\mathbf{x})
$$

for any $\mathbf{x} \in A \otimes_{\ell} B$, so $\theta=\omega$.

We are now ready to prove the main result.

Theorem 2.1. If $\left(G_{A}, u_{A}\right),\left(G_{B}, u_{B}\right)$ are lu-groups and $A, B$ are $M V$-algebras such that $A \simeq \Gamma\left(G_{A}, u_{A}\right)$ and $B \simeq \Gamma\left(G_{B}, u_{B}\right)$ then $A \otimes_{M V} B \simeq \Gamma\left(G_{A} \otimes_{\ell}\right.$ $\left.G_{B}, u_{A} \otimes_{\ell} u_{B}\right)$.

Proof. As before, $A \otimes_{\ell} B$ denotes $\Gamma\left(G_{A} \otimes_{\ell} G_{B}, u_{A} \otimes_{\ell} u_{B}\right)$ and we prove that $A \otimes_{M V} B \simeq A \otimes_{\ell} B$. Let $\gamma_{A, B}: A \times B \rightarrow A \otimes_{\ell} B$ the bimorphism defined as in Lemma 2.2 and $\beta_{A, B}: A \times B \rightarrow A \otimes_{M V} B$ the standard bimorphism of the $\mathrm{MV}$-algebraic tensor product. Using universal property of the tensor product, we get a homomorphism of MV-algebras

$$
\lambda: A \otimes_{M V} B \rightarrow\left[0, \gamma_{A, B}\left(1_{A}, 1_{B}\right)\right] \leq_{i} A \otimes_{\ell} B
$$

such that $\lambda_{A, B} \circ \beta_{A, B}=\gamma_{A, B}$. By Lemma 2.2 there exists an homomorphism of MV-algebras

$$
\delta: A \otimes_{\ell} B \rightarrow\left[0, \beta_{A, B}\left(1_{A}, 1_{B}\right)\right] \leq_{i} A \otimes_{M V} B
$$

such that $\delta \circ \gamma_{A, B}=\beta_{A, B}$.

Then we get:

$$
\begin{aligned}
& (\delta \circ \lambda) \circ \beta_{A, B}=\delta \circ\left(\lambda \circ \beta_{A, B}\right)=\delta \circ \gamma_{A, B}=\beta_{A, B} \\
& (\lambda \circ \delta) \circ \gamma_{A, B}=\lambda \circ\left(\delta \circ \gamma_{A, B}\right)=\lambda \circ \beta_{A, B}=\gamma_{A, B} .
\end{aligned}
$$

Therefore by the universal property of $\beta_{A, B}$ it follows $\delta \circ \lambda=\mathbf{I}_{A \otimes_{M V} B}$, and by the universal property of $\gamma_{A, B}$ it follows $\lambda \circ \delta=\mathbf{I}_{A \otimes_{\ell} B}$, that is the two 
tensor product are MV-algebraic isomorphic, i.e. $\Gamma\left(G_{A}, u_{A}\right) \otimes_{M V} \Gamma\left(G_{B}, u_{B}\right) \simeq$ $\Gamma\left(G_{A} \otimes_{\ell} G_{B}, u_{A} \otimes_{\ell} u_{B}\right)$.

Remark 2.1. We note that, in [14], it is proved that the functor $\Gamma$ commutes with another tensor product denoted $\otimes_{o}$. The tensor product $\otimes_{o}$ of MV-algebras defined in [14] corresponds to the tensor product $\otimes_{o}$ of $\ell$-groups defined in [18] and it is defined using only bilinear functions, instead of bimorphisms and $\ell u$ bilinear functions. In this case, if $A$ and $B$ are MV-algebras, the function $\iota_{A}: A \rightarrow A \otimes_{o} B$ by $\iota_{A}(a)=a \otimes_{o} 1$ is no longer a homomorphism of MValgebras.

Recall that the functor $\Gamma$ maps archimedean $\ell$-groups to semisimple MValgebras. Moreover, one can easily prove that $\Gamma$ also preserve the archimedean tensor product.

Corollary 2.1. If $\left(G_{A}, u_{A}\right), \Gamma\left(G_{B}, u_{B}\right)$ are archimedean lu-groups and $A, B$ are semisimple $M V$-algebras such that $A \simeq \Gamma\left(G_{A}, u_{A}\right)$ and $B \simeq \Gamma\left(G_{B}, u_{B}\right)$ then $A \otimes B \simeq \Gamma\left(G_{A} \otimes_{a} G_{B}, u_{A} \otimes_{a} u_{B}\right)$.

Proof. The proof is similar with the one of Theorem 2.1] The main idea is that $\Gamma\left(G_{A} \otimes_{a} G_{B}, u_{A} \otimes_{a} u_{B}\right)$ satisfy the same universal property that uniquely defines $A \otimes B$, up to isomorphism.

\section{Scalar extension property for semisimple MV- algebras}

In this section we will investigate the scalar extension property for MV-algebras and $\ell u$-groups. First, let us state the property in our context.

$\left(\mathrm{SEP}_{M V}\right)$ If $P$ is a unital PMV-algebra and $A$ is an MV-algebra, then $P \otimes_{M V} A$ has a canonical structure of MV-module over $P$.

$\left(\mathrm{SEP}_{\ell}\right)$ If $R$ is an $\ell$-ring and $G$ is an $\ell$-group, then $R \otimes_{\ell} G$ has a canonical structure of $\ell$-module over $R$.

We summarize the results so far.

Remark 3.1. The scalar extension property is one of the basic property arising from a tensor product, and while it is straightforward in the non-ordered case, with lattice ordered structures it presents some difficulties. 
(1) For MV-algebras, the property $\mathrm{SEP}_{M V}$ is stated in [14, Theorem 4.11], but the proof presents a wrong argument. We note that the structure $P \otimes_{M V} A$ can be endowed with a family of unary operations $\{\alpha\}_{\alpha \in P}$ such that the function $(\alpha, x) \mapsto \alpha x$ is linear in the second argument. The proof of the linearity in the first argument contains a mistake.

(2) For $\ell$-groups, the property $\mathrm{SEP}_{\ell}$ is left as an exercise in [23, Chapter 4.5]. The case $R=\mathbb{R}$, i.e. the $\ell$-modules are Riesz spaces (vector lattices), is considered in [18, Proposition 2.1], but the details are missing.

Our impossibility to correct the proof from [14, Theorem 4.11] and to complete the proof from [18, Proposition 2.1] is related to the fact that the sum of two homomorphisms of $\ell$-groups is not always an homomorphism of $\ell$-groups. We leave this as open problems.

Remark 3.2. Under the assumption that [18, Proposition 2.1] is correct, using Theorem 2.1, one can immediately prove $\left(\mathrm{SEP}_{M V}\right)$ for $P=[0,1]$. This means that for any MV-algebra $A$, the tensor product $[0,1] \otimes_{M V} A$ is a Riesz MValgebra.

Remark 3.3. In [5, Theorem 5], the property $\mathrm{SEP}_{\ell}$ is proved for the archimedean tensor product in the context of Riesz spaces $(R=\mathbb{R})$.

In Theorem 3.1 we prove $\mathrm{SEP}_{M V}$ for the semisimple tensor product allowing $P$ to be an arbitrary unital and semisimple PMV-algebra. Using Theorem 2.1 we get $\mathrm{SEP}_{\ell}$ for archimedean structures allowing $R$ to be an arbitrary archimedean $\ell$-ring with strong unit. In this way we generalize the result from [5].

Remark 3.4. Let $P$ be a PMV-algebra and $S \subseteq P$ a sub PMV-algebra of $P$. It is easily seen that $P$ is a $S$-MV-module such that the external operation coincides with the internal product on $P$.

Lemma 3.1. Let $A \subseteq C(X) \subseteq C(X \times Y)$ be a PMV-algebra. Then the map

$$
\begin{gathered}
\varphi: A \times C(X \times Y) \rightarrow C(X \times Y), \\
\varphi(a, f)(x, y)=a(x) f(x, y) \text { for any } a \in A \text { and } f \in C(X \times Y),
\end{gathered}
$$

defines a structure of $A-M V$-module.

Proof. If $a_{1}+a_{2}$ is defined in $A$, then for any $x \in X$ the partial sum $a_{1}(x)+a_{2}(x)$ is defined and $\left(a_{1}+a_{2}\right) f=a_{1} f+a_{2} f$ if and only if the equality holds for any $x \in X, y \in Y$. Trivially $\left(a_{1}(x)+a_{2}(x)\right) f(x, y)=a_{1}(x) f(x, y)+a_{2}(x) f(x, y)$, 
since the functions are $[0,1]$-valued. In the same way we get all other conditions for an MV-module.

Theorem 3.1. Let $A$ be a unital and semisimple $P M V$-algebra, and $B$ be a semisimple $M V$-algebra. Then $A \otimes B$ is an $A-M V$-module.

Proof. By Theorem 1.1, $A \otimes B=\langle\gamma(a, b) \mid a \in A b \in B\rangle_{M V} \subseteq C(X \times Y)$, with $A \subseteq C(X), B \subseteq C(Y)$.

For any $\alpha \in A$, we define

$$
\omega_{\alpha}: A \times B \rightarrow A \otimes B, \quad \omega_{\alpha}(a, b)(x, y)=\alpha(x) a(x) b(y) .
$$

Since $a(x) b(y)=\gamma(a, b)(x, y)$ and $\alpha(x) \leq 1$, it follows that $\omega_{\alpha}(a, b) \in A \otimes B$. We prove that $\omega_{\alpha}$ is a bimorphism.

Note that $\omega_{\alpha}\left(a_{1} \wedge a_{2}, b\right)(x, y)=\alpha(x)\left[\left(a_{1} \wedge a_{2}\right)(x)\right] b(y)=\alpha(x)\left(a_{1}(x) \wedge\right.$ $\left.a_{2}(x)\right) b(y)$. Since we are in the unital case the product can be distributed on and we get $\wedge$ and $\vee\left[\alpha(x) a_{1}(x) \wedge \alpha(x) a_{2}(x)\right] b(y)=\left[\alpha(x) a_{1}(x) b(y)\right] \wedge\left[\alpha(x) a_{2}(x) b(y)\right]$. In the same way we get the desired property for $\vee$ and on the second component;

If $a_{1}+a_{2}$ defined in $A$, then $\omega_{\alpha}\left(a_{1}+a_{2}, b\right)(x, y)=\alpha(x)\left[a_{1}(x)+a_{2}(x)\right] b(y)$. By the definition of PMV-algebra, we get the desired conclusion.

Therefore, applying the universal property, there exists a homomorphism of MV-algebras

$$
\Omega_{\alpha}: A \otimes B \rightarrow\left[0, \omega_{\alpha}(1,1)\right] \leq_{i} A \otimes B
$$

We remark that $\omega_{\alpha}(1,1)(x, y)=\alpha(x) \mathbf{1}(x) \mathbf{1}(y)=\alpha(x)$. Moreover, $\Omega_{\alpha}: A \otimes B \rightarrow$ $\left[0, \omega_{\alpha}(1,1)\right] \leq_{i} A \otimes B \subseteq C(X \times Y)$.

By Lemma 3.1. $C(X \times Y)$ is $A$-MV-module with external operation $\varphi: A \times$ $C(X \times Y) \rightarrow C(X \times Y)$. We fix $\alpha \in A$, and we define

$$
\theta_{\alpha}: C(X \times Y) \rightarrow C(X \times Y), \quad \theta_{\alpha}(f)=\varphi(\alpha, f) .
$$

Since $C(X \times Y)$ is $A$-MV-module, $\theta_{\alpha}$ is linear and $\theta_{\alpha}(f) \leq f$. It follows that $\theta_{\alpha}\left(f_{1}\right) \wedge f_{2}=0$ whenever $f_{1} \wedge f_{2}=0$. Note that such functions are called $f$-operators in [15]. By [15, Proposition 5.9], $\theta_{\alpha}$ is an MV-algebra morphism

$$
\theta_{\alpha}: C(X \times Y) \rightarrow\left[0, \theta_{\alpha}(1)\right] \leq_{i} C(X \times Y) .
$$

Moreover, $\theta_{\alpha}(1)(x, y)=\varphi(\alpha, 1)(x, y)=\alpha(x)$.

Then we have

$$
\begin{aligned}
& \Omega_{\alpha}: A \otimes B \rightarrow\left[0, \omega_{\alpha}(1,1)\right] \leq_{i} A \otimes B \subseteq C(X \times Y) \text { and } \\
& \left.\theta_{\alpha}\right|_{A \otimes B}: A \otimes B \rightarrow\left[0, \theta_{\alpha}(1)\right] \leq_{i} C(X \times Y) .
\end{aligned}
$$

Since $\omega_{\alpha}(1,1)=\theta_{\alpha}(1)$, by [14, Lemma 2.3], 


$$
\left[0, \omega_{\alpha}(1,1)\right]_{A \otimes B} \subseteq\left[0, \theta_{\alpha}(1)\right] \leq_{i} C(X \times Y) .
$$

Therefore $\Omega_{\alpha}$ and $\left.\theta_{\alpha}\right|_{A \otimes B}$ are both maps from $A \otimes B$ in $\left[0, \theta_{\alpha}(1)\right] \leq_{i} C(X \times Y)$, and by universal property they are the same map if they coincide on generators, but this is trivially true, since for any $a \in A, b \in B, x \in X, y \in Y$

$$
\Omega_{\alpha}(\gamma(a, b))(x, y)=\alpha(x) a(x) b(y)=\varphi(\alpha, \gamma(a, b))(x, y)=\theta_{\alpha}(\gamma(a, b))(x, y) .
$$

As result, we have $A \otimes B$ included in $C(X \times Y)$ as MV-algebra, and two families of linear functions:

$$
\left\{\Omega_{\alpha}: A \otimes B \rightarrow A \otimes B\right\}_{\alpha \in A} ; \quad\left\{\theta_{\alpha}: C(X \times Y) \rightarrow C(X \times Y)\right\}_{\alpha \in A}
$$

such that $\left.\theta_{\alpha}\right|_{A \otimes B}=\Omega_{\alpha}$, and $\left(C(X \times Y),\left\{\theta_{\alpha}\right\}_{\alpha \in A}\right)$ is an $A$-MV-module.

Since for any $\alpha \in A$ we have $\left.\theta_{\alpha}\right|_{A \otimes B}(A \otimes B)=\Omega_{\alpha}(A \otimes B) \subseteq A \otimes B, A \otimes B$ is closed to the scalar product, and $\left(A \otimes B,\left\{\left.\theta_{\alpha}\right|_{A \otimes B}\right\}_{\alpha \in A}\right)$ is an sub MV-module of $C(X \times Y)$.

By Theorem 2.1 and Theorem 3.1 we immediately get the following.

Theorem 3.2. If $R$ is a unital and archimedean $\ell u$-ring and $G$ is an archimedean

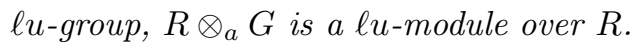

In the sequel, we apply use $\mathrm{SEP}_{M V}$ in order to establish connections between MV-algebras and Riesz MV-algebras.

Proposition 3.1. Let $A$ be a semisimple Riesz $M V$-algebra, and $B$ be a semisimple $M V$-algebra. Then $A \otimes B$ is an Riesz $M V$-algebra. In particular, $[0,1] \otimes B$ is a Riesz MV-algebra.

Proof. The proof is similar with the one of Theorem 3.1. The starting bimorphism will be

$\omega_{\alpha}: A \times B \rightarrow A \otimes B, \omega_{\alpha}(a, b)=\alpha a(x) b(y)$ where $\alpha \in[0,1]$.

By Theorem 2.1 and Proposition 3.1 we immediately get the following.

Proposition 3.2. Let $V$ be an archimedean Riesz Space with strong unit and $G$ be an archimedean lu-group. Then $V \otimes_{a} G$ is an archimedean Riesz Space with strong unit. In particular, $\mathbb{R} \otimes_{a} G$ is a Riesz Space.

Remark 3.5. Let $B$ be a semisimple MV-algebra and $\iota_{B}: B \rightarrow[0,1] \otimes B$ the canonical embedding. Since $[0,1] \otimes B$ is generated, as a Riesz MV-algebra, by $\iota_{B}(B)$ it follows by [12, Corollary 4.2$]$, that $[0,1] \otimes A$ is, up to isomorphism, the Riesz MV-algebra hull of $B$. One can see [12] for more details. 
Corollary 3.1. Let $B$ be a semisimple $M V$-algebra. For any semisimple Riesz $M V$-algebra $V$ and for any homomorphism of $M V$-algebras $f: B \rightarrow \mathcal{U}_{\mathbb{R}}(V)$ there is a unique homomorphism of Riesz $M V$-algebras $\widetilde{f}:[0,1] \otimes B \rightarrow V$ such that $\tilde{f} \circ \iota_{B}=f$.

Proof. Define $\beta_{f}:[0,1] \times B \rightarrow V$ by $\beta(\alpha, x)=\alpha f(x)$ for any $\alpha \in[0,1]$ and $x \in B$ and use the universal property of the tensor product. We note that any MV-algebra homomorphism between Riesz MV-algebras preserves the scalar multiplication, so it is a morphism of Riesz MV-algebras [11, Corollary 3.11].

Assume that $\mathbf{M V}_{\mathbf{s s}}$ is the full subcategory of semisimple MV-algebras and $\mathbf{R M V}_{\mathbf{s s}}$ is the full subcategory of semisimple Riesz MV-algebras. Hence we define a functor $\mathcal{T}_{\otimes}: \mathbf{M} \mathbf{V}_{\mathbf{s s}} \rightarrow \mathbf{R} \mathbf{M} \mathbf{V}_{\mathbf{s s}}$ by

$\mathcal{T}_{\otimes}(B)=[0,1] \otimes B$ for any semsimple MV-algebra $B$ and

$\mathcal{T}_{\otimes}(f)=\tilde{f}$ for any homomorphism of MV-algebras $f: A \rightarrow B$, where $\tilde{f}$ : $[0,1] \otimes A \rightarrow[0,1] \otimes B$ is the unique Riesz MV-algebra homomorphism such that $\tilde{f} \circ \iota_{A}=\iota_{B} \circ f$ (which exists by Corollary 3.1).

Corollary 3.2. Under the above hypothesis, $\left(\mathcal{T}_{\otimes}, \mathcal{U}_{\mathbb{R}}\right)$ is an adjoint pair.

Proof. It is straightforward. Using a different construction of the Riesz hull, this result is proved in [12].

\section{The categorical adjunction between semisimple $\mathrm{PMV}$-algebras and semisimple $f \mathrm{MV}$-algebras}

In the sequel, using the scalar extension property, we define an adjunction between the category of semisimple and unital PMV-algebras and the category of unital and semisimple $f \mathrm{MV}$-algebras.

As a preliminary step, we prove the following theorem.

Proposition 4.1. Let $A, B$ be unital and semisimple PMV-algebras. Then $A \otimes B$ is a unital and semisimple PMV-algebra.

Proof. By Theorem 1.1, $A \otimes B=\langle\gamma(a, b) \mid a \in A b \in B\rangle_{M V} \subseteq C(X \times Y)$ with $A \subseteq C(X), B \subseteq C(Y)$, as MV-algebra.

For any $c \in A \otimes B$, we define $\omega_{c}: A \times B \rightarrow A \otimes B, \omega_{c}(a, b)(x, y)=c(x, y) a(x) b(y)$. Since $a(x) b(y)=\gamma(a, b)(x, y)$ and $c(x, y) \leq 1, \omega_{\alpha}(a, b) \in A \otimes B$ and it is a 
bimorphism, likewise in the proof of Theorem 3.1. Therefore, applying the universal property, there exist a map

$$
\Omega_{c}: A \otimes B \rightarrow\left[0, \omega_{c}(1,1)\right] \leq_{i} A \otimes B .
$$

Again, $\omega_{c}(1,1)(x, y)=c(x, y) \mathbf{1}(x) \mathbf{1}(y)=c(x, y)$ and $\Omega_{c}: A \otimes B \rightarrow\left[0, \omega_{c}(1,1)\right] \leq_{i}$ $A \otimes B \subseteq C(X \times Y)$.

It is straightforward that $C(X \times Y)$ is PMV-algebra with internal product * : $C(X \times Y) \times C(X \times Y) \rightarrow C(X \times Y)$ defined component-wise. We fix $c \in A \otimes B$, and we define

$$
\theta_{c}: C(X \times X) \rightarrow C(X \times Y), \quad \theta_{c}(f)=c * f .
$$

It is easy to prove, since $C(X \times Y)$ is unital PMV-algebra, that $\theta_{c}$ is linear and $\theta_{c}(f) \leq f$. And again like in the proof of Theorem 3.1. $\theta_{c}: C(X \times$ $Y) \rightarrow\left[0, \theta_{c}(1)\right] \leq_{i} C(X \times Y)$ is an homomorphism of MV-algebras. Moreover, $\theta_{c}(1)(x, y)=(c * 1)(x, y)=c(x, y)$.

Then we have

$$
\begin{aligned}
& \Omega_{c}: A \otimes B \rightarrow\left[0, \omega_{c}(1,1)\right] \leq_{i} A \otimes B \subseteq C(X \times Y) \text { and } \\
& \left.\theta_{c}\right|_{A \otimes B}: A \otimes B \rightarrow\left[0, \theta_{c}(1)\right] \leq_{i} C(X \times Y) .
\end{aligned}
$$

Since $\omega_{c}(1,1)=\theta_{c}(1)$, we get $\left[0, \omega_{c}(1,1)\right]_{A \otimes B} \subseteq\left[0, \theta_{c}(1)\right] \leq_{i} C(X \times Y)$, and the conclusion follows like in Theorem 3.1

Finally, it can be easily seen that the unit in $A \otimes B$ is the element $1_{A} \otimes 1_{B}$. Then $A \otimes B$ is unital, therefore it is semisimple as PMV-algebra.

Theorem 4.1. Let $R$ be a fMV-algebra and $P$ be a unital and semisimple $P M V$ algebra. Then $R \otimes P$ is a unital and semisimple $f M V$-algebra.

Proof. By construction $R \otimes P$ is a semisimple MV-algebra, by Corollary 3.1 . $R \otimes P$ is a Riesz MV-algebra and by Proposition 4.1 it is a unital and semisimple PMV-algebra. Moreover, by the construction of the product and the scalar operation as the usual product and scalar operation between functions given in Proposition 4.1 and Theorem 3.1, the associativity law between products is satisfied since it holds in any $C(X)$. It follows that $R \otimes P$ is a unital and semisimple $f \mathrm{MV}$-algebra.

Proposition 4.2. Let $A$ be a unital and semisimple PMV-algebra. For any unital and semisimple $f M V$-algebra $M$ and for any homomorphism of PMValgebras $f: A \rightarrow \mathcal{U}_{\mathbb{R}}(M)$ there is a unique homomorphism of $f M V$-algebras $\tilde{f}:[0,1] \otimes A \rightarrow M$ such that $\tilde{f} \circ \iota_{A}=f$, where $\iota_{A}: A \rightarrow[0,1] \otimes A$ is the embedding in the tensor product. 
Proof. By Corollary [3.1, there exists a homomorphism of Riesz MV-algebras $\tilde{f}:[0,1] \otimes A \rightarrow \mathcal{U}_{(\cdot)}(M)$ such that $\tilde{f} \circ \iota_{A}=f$. Since $[0,1] \otimes A$ is a $f M V$-algebra by Theorem 4.1, $\tilde{f}$ is a homomorphism of Riesz MV-algebras between unital and semisimple $f \mathrm{MV}$-algebras. By [17, Proposition 3.2] $\widetilde{f}$ is a homomorphism of $f \mathrm{MV}$-algebras.

Proposition 4.3. Let $P_{1}$ and $P_{2}$ be semisimple and unital $P M V$-algebras, and $h: P_{1} \rightarrow P_{2}$ a homomorphism of $P M V$-algebras. Then there exists a unique $h^{\sharp}:[0,1] \otimes P_{1} \rightarrow[0,1] \otimes P_{2}$ homomorphism of $f M V$-algebras such that $h^{\sharp} \circ \iota_{1}=$ $\iota_{2} \circ h$, where $\iota_{i}: P_{i} \rightarrow[0,1] \otimes P_{i}$ for $i=1,2$ are the natural embeddings.

Proof. It is straightforward by Proposition 4.2, with $f=\iota_{2} \circ h$.

Let $\mathbf{u P M V}$ ss be the full subcategory of semisimple and unital PMV-algebras with homomorphism of PMV-algebras and let $\mathbf{u f M V} \mathbf{V}_{\mathbf{s s}}$ be the full subcategory of semisimple and unital $f \mathrm{MV}$-algebras with homomorphism of $f \mathrm{MV}$-algebras. We define a functor $\mathcal{F}_{\otimes}: \mathbf{u P M V}_{\mathbf{s s}} \rightarrow \mathbf{u f M V} \mathbf{V}_{\mathbf{s s}}$ as follows

(i) for any $P \in \mathbf{u P M V} \mathbf{V}_{\mathbf{s s}}, \mathcal{F}_{\otimes}(P)$ is $[0,1] \otimes P$. By Theorem 4.1 it is a unital, commutative and semisimple $f \mathrm{MV}$-algebra.

(ii) for any homomorphism of PMV-algebras $h: P_{1} \rightarrow P_{2}, \mathcal{T}(h)$ is the homomorphism of $f \mathrm{MV}$-algebras $h^{\sharp}$ defined in Proposition 4.3

From $\mathbf{u f M V} \mathbf{V}_{\mathbf{s s}}$ to $\mathbf{u P M V _ { \mathbf { s s } }}$ we have the usual forgetful functor $\mathcal{U}_{\mathbb{R}}$.

Lemma 4.1. $\mathcal{F}_{\otimes}$ is a functor.

Proof. Let $P_{1}, P_{2}, P_{3}$ be PMV-algebras and $h: P_{1} \rightarrow P_{2}$ and $g: P_{2} \rightarrow P_{3}$ PMValgebras homomorphisms. Let $\iota_{1}, \iota_{2}$ and $\iota_{3}$ be the embeddings of $P_{1}, P_{2}, P_{3}$ in $[0,1] \otimes P_{1},[0,1] \otimes P_{2}$ and $[0,1] \otimes P_{3}$ respectively. Then

$$
\left(g^{\sharp} \circ h^{\sharp}\right) \circ \iota_{1}=g^{\sharp} \circ\left(h^{\sharp} \circ \iota_{1}\right)=g^{\sharp} \circ\left(\iota_{2} \circ h\right)=\left(g^{\sharp} \circ \iota_{2}\right) \circ h=\iota_{3} \circ(g \circ h)
$$

and by Proposition 4.3 we get $g^{\sharp} \circ h^{\sharp}=(g \circ h)^{\sharp}$.

Lemma 4.2. The maps $\left\{\iota_{A}\right\}_{A \in \mathbf{u P M V}}$ are a natural transformation between the identity functor on $\mathbf{u P M V} \mathbf{s s}$ and $\mathbf{u} \mathbf{M} \mathbf{M V}_{\mathbf{s s}}$.

Proof. Let $P_{1}, P_{2} \in \mathbf{u P M V} \mathbf{V}_{\mathbf{s s}}$ and let $h: P_{1} \rightarrow P_{2}$ be a homomorphism of PMValgebras. We need to prove that $\mathcal{U}_{\mathbb{R}} \mathcal{F}_{\otimes}(h) \circ \iota_{1}=\iota_{2} \circ h$. Since $\mathcal{U}_{\mathbb{R}} \mathcal{F}_{\otimes}(h)=h^{\sharp}$ the result follows from Proposition 4.3 . 
Theorem 4.2. The functors $\mathcal{F}_{\otimes}$ and $\mathcal{U}_{\mathbb{R}}$ are adjoint functors.

Proof. In order to prove that $\mathcal{F}_{\otimes}$ is left adjoint functor of $\mathcal{U}_{\mathbb{R}}$, we need to prove that for any semisimple and unital $f \mathrm{MV}$-algebra $A$ and any homomorphism of PMV-algebras $f: P \rightarrow \mathcal{U}_{\mathbb{R}}(A)$, with $P \in \mathbf{u} \mathbf{P M V}$ ss, there exists a homomorphism of $f$ MV-algebras $f^{\sharp}: \mathcal{T}(P) \rightarrow A$ such that $\mathcal{U}_{\mathbb{R}}\left(f^{\sharp}\right) \circ \iota_{P}=f$. This follows from Proposition 4.2

Results in the previous sections can transferred to $\ell u$-groups and all related structures. We remark that Proposition 4.1. Theorem 4.1 and Corollary 2.1 entail the following.

Proposition 4.4. (i) If $R$ and $S$ are unital and archimedean lu-rings, $R \otimes_{a} S$ is a unital and archimedean $\ell u$-ring.

(ii) If $V$ is a unital and archimedean fu-algebra and $R$ is a unital and archimedean $\ell u$-ring, $V \otimes_{a} R$ is a unital and archimedean fu-algebra.

Proof. It is straightforward by Theorem 4.1, Corollary 2.1 and the categorical equivalence.

\section{Conclusions}

By categorical equivalence, the adjunctions $\left(\mathcal{T}_{\otimes}, \mathcal{U}_{\mathbb{R}}\right)$ and $\left(\mathcal{F}_{\otimes}, \mathcal{U}_{\mathbb{R}}\right)$ naturally transfer to lattice-ordered structure. We denote by $\mathbf{a u G}_{\mathbf{a}}$ the category of archimedean $\ell u$-groups; $\mathbf{u R}_{\mathbf{a}}$ the category of archimedean and unital $\ell u$-rings; $\mathbf{u R S}_{\mathbf{a}}$ the category of archimedean Riesz Spaces with strong unit; fuAlga the category of archimedean and unital $f u$-algebras.

Applying the inverse of $\Gamma$ and $\Gamma_{\mathbb{R}},\left(\mathcal{T}_{\otimes}, \mathcal{U}_{\mathbb{R}}\right)$ extends to $\left(\mathcal{T}_{\otimes a}, \mathcal{U}_{\ell \mathbb{R}}\right)$. This is an adjunction between $\mathbf{a u G}_{\mathbf{a}}$ and $\mathbf{u R S}_{\mathbf{a}}$.

Applying the converses of the functors $\Gamma_{(\cdot)}$ and $\Gamma_{f},\left(\mathcal{F}_{\otimes}, \mathcal{U}_{\mathbb{R}}\right)$ extends to $\left(\mathcal{F}_{\otimes a}, \mathcal{U}_{\ell \mathbb{R}}\right)$. This is an adjunction between $\mathbf{u R}_{\mathbf{a}}$ and $\mathbf{f u A l g} \mathbf{a}$.

In the following we summarize or results.

Theorem 5.1. The following diagrams are commutative: 

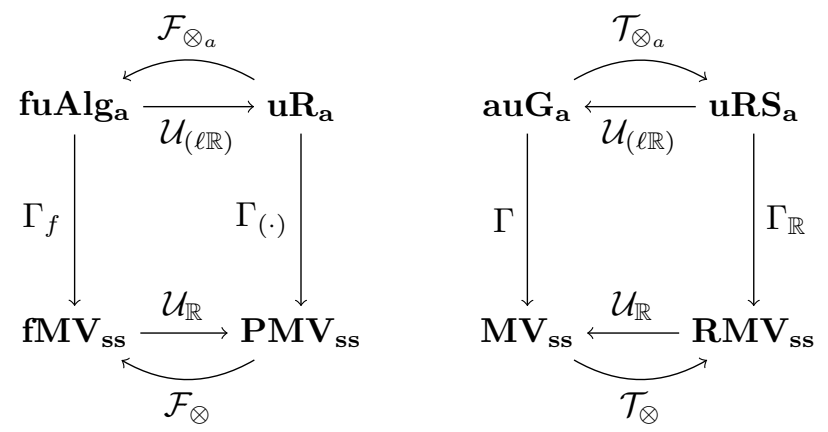

Proof. It is a direct consequence of Theorem 2.1 .

Recall that MV-algebras were defined as the algebraic structures corresponding to Eukasiewicz $\infty$-valued logic. Even if their algebraic theory is relevant in itself as proved by [8, 22, it has been developed in strong connection with the associated logical system. The same holds for PMV-algebras [19], their theory has its origins in the problem of enriching Łukasiewicz logic with a binary conector whose interpretation in $[0,1]$ is the natural product. Logical systems were also defined for Riesz MV-algebras [11] and $f \mathrm{MV}$-algebras [17]. Note that for PMV-algebras and $f \mathrm{MV}$-algebras the logical systems are developed only for particular suitable subclasses. One important link between logic and algebra in all these cases is the Lindenbaum-Tarski algebra, which is the free algebra generated by the set of propositional variables. Due to the fact that the free algebras are semisimple structures, the scalar extension property allows us to connect the free structures as in Proposition 5.1

For a nonempty set $X$, let Free $_{M V}(X)$ and $\operatorname{Free}_{R M V}(X)$ be the free MValgebra and, respectively, the free Riesz MV-algebras generated by $X$. Let Free $_{P M V}(X)$ be the free PMV-algebra in $H S P\left([0,1]_{P M V}\right)$, the variety of PMValgebras generated by $[0,1]$. Similarly, let $\operatorname{Free}_{f M V}(X)$ be the free $f \mathrm{MV}$-algebra in $\operatorname{HSP}\left([0,1]_{f M V}\right)$, the variety of $f \mathrm{MV}$-algebras generated by $[0,1]$. See more details in [8, 19, 11, 17].

Proposition 5.1. For any nonempty set $X$, the following hold:

(i) $\operatorname{Free}_{R M V}(X) \simeq[0,1] \otimes$ Free $_{M V}(X)$,

(ii) $\operatorname{Free}_{f M V}(X) \simeq[0,1] \otimes$ Free $_{P M V}(X)$.

Proof. (i) follows by Remark 3.5 and [12, Proposition 4.1]; it can also be proved directly, similarly with (ii). 
(ii) Assume $V$ is a $f \mathrm{MV}$-algebra and $f: X \rightarrow V$ is a function. Hence there is a unique homomorphism of PMV-algebras $f^{\#}:$ Free $_{P M V}(X) \rightarrow \mathcal{U}_{\mathbb{R}}(V)$ which

extends $f$. By Proposition 4.2 there exists a homomorphism of $f \mathrm{MV}$-algebras $\tilde{f}:[0,1] \otimes \operatorname{Free}_{P M V}(X) \rightarrow V$ such that $\tilde{f} \circ \iota_{F_{r e e} e_{P V(X)}}=f^{\#}$, so $\widetilde{f}(1 \otimes x)=$ $f(x)$ for any $x \in X$. The uniqueness of $\tilde{f}$ is a consequence of the uniqueness of $f^{\#}$. Since $\iota_{F r e e_{P M V(X)}}$ is an embedding we have $X \simeq\{1 \otimes x \mid x \in X\}$ so $[0,1] \otimes \operatorname{Free}_{P M V}(X)$ satisfies the universal property that uniquely defines Free $_{f M V}(X)$.

\section{References}

[1] Ball R.N., Georgescu G., Leuştean I., Cauchy completions of MV-algebras, Algebra Universalis 47(4) (2002) 367-407.

[2] Bigard A., Keimel K., Wolfenstein S., Groupes et anneaux réticulés, Lectures Notes in Mathematics Vol. 608 (1977), Springer-Verlag.

[3] Birkhoff G., Lattice Theory, AMS Coll. Publ. 25 3rd Ed. 1973.

[4] Birkhoff G., Pierce R.S., Lattice-ordered rings, An. Acad. Brasil. Cienc. 28 (1956) 41-69.

[5] Buskes G.J.H.M., Van Rooij A.C.M., The Archimedean $\ell$-group tensor product, Order 10 (1993) 93-102.

[6] Chang C.C., Algebraic analysis of many valued logics, Trans. Amer. Math. Soc. 88 (1958) 467-490.

[7] Chang C.C., A new proof of the completeness of the Eukasiewicz axioms, Transactions of the American Mathematical Society 93 (1959), 74-80.

[8] Cignoli R., D'Ottaviano I.M.L., Mundici D., Algebraic foundation of many valued Reasoning, Kluver Academc Publ Dordrecht 2000.

[9] Di Nola A., Dvurecenskij A., Product $M V$-algebras, Multiple-Valued Logics 6 (2001) 193-215.

[10] Di Nola A., Flondor P., Leustean I., MV-modules, Journal of Algebra 267(1) (2003) 21-40. 
[11] Di Nola A., Leustean I., Lukasiewicz logic and Riesz Spaces, Soft Comp. , to appear.

[12] Diaconescu D., Leuştean I., The Riesz hull of a semisimple MV-algebra, Mathematica Slovaca (special issue in honor of Antonio Di Nola), in print.

[13] Flaminio T., Montagna F., private communication.

[14] Flondor P., Leuştean I., Tensor Product of MV-algebras, Soft Computing 7 (2003) 446-457.

[15] Flondor P., Leuştean I., MV-algebras with operator (the commutative and non-commutative case), Discrete Mathematics 274 (2004) 41-76.

[16] Fremlin D.H., Tensor Product of Archimedean Vector Lattices, American Journal of Math. 94(3) (1972) 777-798.

[17] Lapenta S., Leuştean I., Towards Pierce-Birkhoff conjecture via MValgebras, submitted.

[18] Martinez J., Tensor Product of partially ordered groups, Pac. J. Math. 41 (1972) 771-789.

[19] Montagna F., An algebraic approach to Propositional Fuzzy Logic, Journal of Logic, Language and Information 9 (2000) 91-124.

[20] Mundici D., Interpretation of $A C F^{*}$-algebras in Eukasiewicz sentential calculus, J. Funct. Anal. 65 (1986) 15-63.

[21] Mundici D., Tensor products and the Loomis-Sikorski theorem for MValgebras, Advanced in Applied Mathematics 22 (1999) 227-248.

[22] Mundici D., Advances in Eukasiewicz calculus and MV-algebras, Trends in Logic 35 Springer 2011.

[23] Steinberg S.A., Lattice-Ordered Rings and Modules, Springer, 2010.

[24] Zaneen A.C., Riesz Space II, North Holland, Amsterdam 1983. 